\title{
LA RÚBRICA COMO ELEMENTO DE EVALUACIÓN: APLICACIÓN Y ANÁLISIS EN DISCIPLINAS HUMANÍSTICAS.
}

\author{
THE RUBRIC AS AN ELEMENT OF EVALUATION: APPLICATION AND ANALYSIS IN HUMANISTIC \\ DISCIPLINES.
}

\author{
Rocío Velasco García*, Manuel Pérez Lozano, \\ Antonio José Díaz Rodríguez, María Ángeles Jordano Barbudo, \\ Carlos Márquez Moreno, Enrique Soria Mesa, José Luis Sanchidrián Torti, \\ Rafael Bonilla Cerezo, Dámaris Romero Gónzález, Israel Muñoz Gallarte, \\ Luis Salas Almela, Blas Sánchez Dueñas, Josefa Mata Torres, \\ Alicia Carrillo Calderero, Savina de Cavi, José María García Ríos. \\ *rocio.velasco@uco.es
}

Received: 30/06/2017_Accepted: 30/10/2018

\section{Resumen}

El presente artículo describe el desarrollo de un Proyecto Docente sobre la difusión, implementación y evaluación de la herramienta de la rúbrica de evaluación en asignaturas humanísticas, así como el fomento de la comunicación entre alumnado y profesorado en relación con los procesos e instrumentos de evaluación y aprendizaje por tareas, en base a los objetivos de evaluación por competencias. Las acciones han ido dirigidas a 18 asignaturas, (todas de la rama de humanidades), y en la que han intervenido profesorado de 4 departamentos de la Facultad de Filosofía y Letras de la Universidad de Córdoba. La aplicación de la rúbrica de evaluación en las diversas asignaturas relacionadas con el proyecto afectó a más de 850 alumnos de 5 titulaciones diferentes. Tras un proceso de estudio, elaboración, revisión e implementación, se llegó a una actividad evaluación dirigida tanto a el alumnado como al profesorado, que se resume en un alto grado de satisfacción por ambas partes y en la continuada aplicación de esta herramienta en cursos posteriores.

Palabras clave: rúbrica, evaluación, competencias, humanidades.

\section{Abstract}

This article describes the development of a Teaching Project on the dissemination, implementation and evaluation of the tool of the rubric of evaluation in humanistic subjects, as well as the promotion of the communication between students and teachers in relation to the processes and instruments of evaluation and learning by tasks, based on the objectives of evaluation by competences. The actions have been directed to 18 subjects, (all of them from the branches of humanities), and in which teachers from 4 departments of the Faculty of Philosophy and Letters of the University of Córdoba have intervened. The application of the rubric of evaluation in the various subjects related to the project affected more than 850 students of 5 different degrees. After a process of study, elaboration, review and implementation, an evaluation activity was reached aimed at both students and teachers, which is summarized in a high degree of satisfaction by both parties and in the continued application of this tool in subsequent courses .

Keywords: rubric, evaluation, competences, humanities.

\section{INTRODUCCIÓN}

La rúbrica de evaluación permite que el alumnado tenga gran cantidad de información respecto a los criterios de evaluación del profesorado, fomentando el autoaprendizaje, la autoevaluación y la responsabilidad, que en función de los criterios propuestos pueden revisar sus trabajos antes de ser entregados.

La propuesta de este proyecto vino motivada por dos hechos relevantes que podríamos resumir en: los óptimos resultados pervios en el proceso de enseñanza-aprendizaje y comunicación entre docente-discente, así como en las calificaciones finales del alumnado, por parte de la coordinadora del proyecto y su alumnado en cursos académicos anteriores. Y el interés por parte de un gran número de docentes de titulaciones humanísticas, por conocer y aplicar la herramienta de "rúbrica de evaluación", tras conversaciones previas mantenidas.

La herramienta en sí no es una novedad, pero sí el hecho de difundirla entre los compañeros docentes de disciplinas humanísticas, el hecho de adaptarlas a las necesidades de las titulaciones, cursos y asignaturas de dichas disciplinas y el hecho de evaluar los resultados obtenidos (pues que sepamos, pese a la amplia bibliografía sobre el asunto, no se han elaborado encuestas de satisfacción al alumnado ni al profesorado que implementa esta herramienta). Uno de los puntos a destacar en este proyecto, ha sido el hecho de compartir los conocimientos y experiencia adquiridos previamente con el resto de 
participantes, con el fin de llevarlo a las aulas y mejorar así la productividad de nuestro alumnado, así como la comunicación entre docente-discente.

La aplicación de esta herramienta ha permitido dejar clara las expectativas de los profesores respecto del alumnado, así como aportar seguridad al discente sobre cómo alcanzar lo que el profesor espera de él/ella. De este modo el alumnado ha conseguido tener más información sobre sus fortalezas y debilidades en la asignatura, por lo que estaríamos hablando de "retroalimentación" fomentando de este modo el aprendizaje y la autoevaluación, tal y como nos dice John Biggs [1], Amparo Fernández [3] o María Alejandra Zazueta Hernández y Luis Fernando [4] quienes, entre otros, tratan en sus publicaciones sobre los procesos de alimentación y retroalimentación y los sistemas o recursos de evaluación como parte del aprendizaje. A su vez, se ha promovido la responsabilidad del alumnado en función a los criterios propuestos por el profesorado, en la que han podido revisar sus trabajos antes de ser entregados al docente.

Por parte del profesorado, esta herramienta les ha permitido incrementar la objetividad del proceso evaluativo, ajustándola a la exigencia de la evaluación por competencias, pues no podemos perder de vista la importancia de la mismas tal y como recoge la obra de Elena Cano en sus primeros capítulos [2]. Igualmente, les ha facilitado las instrucciones sobre las calificaciones antes de su revisión, y les ha ayudado a agilizar el proceso de corrección. Por otra parte, ello implicó que el profesorado utilizara parámetros de puntuación en la evaluación del desempeño del alumnado, describiendo las características específicas de una tarea en varios niveles de rendimiento. La finalidad de su aplicación fue clarificar lo que se espera del trabajo del alumno/a, valorar su ejecución y facilitar la proporción de retroalimentación.

\section{OBJETIVOS}

Los objetivos planteados en el proyecto fueron los siguientes:

A. Difundir la herramienta de rúbrica de evaluación entre el profesorado de disciplinas humanísticas como mejora del proceso enseñanza-aprendizaje y los resultados académicos del alumnado.

B. Adaptar y aplicar la rúbrica de evaluación en el sistema de evaluación docente en cursos, grados y asignaturas de distinto contenido de diversas disciplinas humanísticas.

C. Analizar y evaluar la herramienta de rúbrica de evaluación entre los participantes del presente proyecto y el alumnado al que se dirige.

D. Implantar dicha herramienta en la elaboración de futuras guías docentes por parte del profesorado participante en este proyecto.

\section{METODOLOGÍA}

Se estableció la duración del presente proyecto desde el 11 de septiembre de 2017 (comienzo de las clases) hasta principios el mismo día 30 de junio de 2018, última fecha de examen de una de las asignaturas implicadas en este proyecto. Con un total de 18 asignaturas implicadas, repartidas entre primer y segundo cuatrimestre, de distintos niveles que iban desde el $1^{\circ}$ al $4^{\circ}$ curso, en diversas disciplinas humanísticas como Grado de Historia, Gestión Cultural, Historia del Arte, itinerario conjunto del Grado de Historia e Historia del Arte, Turismo y Filología Hispánica, este proyecto ha revertido en más de 850 alumnos con 16 docentes como participantes en el mismo, más la coordinadora que suscribe esta memoria, quien también ha participado activamente en la implantación de la rúbrica de evaluación.

En lo referente a la planificación, se establecieron previamente una serie de sesiones informativas y formativas por parte de la coordinadora, acompañadas de otras de trabajo en equipo, para terminar con una puesta en común sobre los resultados obtenidos. Dichas sesiones se desarrollaron de la siguiente manera:

1. En primera instancia, se convocó al profesorado participante a una reunión divulgativa y didáctica, en la primera semana de inicio del curso, viernes 15 de septiembre, aun cuando no había resolución de aprobación del proyecto. Se hacía necesario empezar a trabajar lo antes posible para informar y formar al profesorado, y por consiguiente, al alumnado de los diferentes grados y asignaturas implicadas y correspondientes al primer cuatrimestre. Aquella reunión fue dirigida por la coordinadora del proyecto, con el fin de aleccionar y mostrar a los componentes del grupo en qué consisten las rúbricas de evaluación, las ventajas de su utilización en los sistemas de evaluación y mejora de los procesos de enseñanzaaprendizaje, así como las distintas posibilidades de configuración de la misma, acompañada de ejemplos y experiencias previas de la coordinadora. La sesión fue acompañada de una presentación, ejemplos de rúbricas de evaluación elaborados 
previa experiencia y formación de la coordiandora (imagen 1) tras cursas los cursos de formación del profesorado universitario de la Universidad de Córdoba.

Seguidamente, y en el desarrollo de la misma reunión, se debatió sobre indicadores y parámetros conjuntos que pudieran servir de ayuda a la hora de elaborar y adaptar las tablas de rúbricas de evaluación del profesorado para sus asignaturas, atendiendo a las competencias propias de cada asignatura, según los criterios de evaluación establecidos ya en las guías docentes del curso académico en el que se desarrollará el proyecto.

2. En una segunda reunión de trabajo, muy próxima a la anterior, se volvió a convocar al profesorado participante que presentó en ella las distintas rúbricas de evaluación elaboradas y diseñadas, con el fin de ponerlas en común y hacer las modificaciones o rectificaciones necesarias para su definición final. La proximidad de esta segunda reunión de trabajo con respecto a la primera vino motivada por el hecho de que aquellos profesores que impartían sus asignaturas en el primer cuatrimestre tuvieran tiempo suficiente de hacer las correspondientes adaptaciones, ultimar y difundirla entre su alumnado.

3. Una vez el profesorado tuvo definida su rúbrica de evaluación para la asignatura en la que impartía docencia, hizo difusión de ella a su alumnado, mediante la plataforma Moodle en el Aula Virtual de las correspondientes asignaturas indicadas en el proyecto. Se estableció este sistema de comunicación con el alumnado matriculado en dichas materias, con el fin de que tengan pleno acceso durante el desarrollo de las asignaturas pertinentes, favoreciendo así la comunicación entre docentediscente. Igualmente, los docentes explicaron en sus respectivas aulas, la composición y finalidad de sus rúbricas para solventar cualquier duda del alumnado.

4. Tras la revisión de los instrumentos de evaluación de cada asignatura, desde la coordinación del presente proyecto se hizo entrega de unas encuestas de elaboración propia, para ser completadas tanto por el profesorado como por el alumnado participante, con el fin de observar el grado de efectividad.

5. Tuvieron lugar otras reuniones, tanto presenciales como virtuales, sobre cuestiones o dudas surgidas durante la elaboración personal de la rúbrica. Surgieron cuestiones muy interesantes sobre las ventajas y desventajas en el proceso de elaboración que revertirían en el de ejecución.

6. Una vez concluido el primer cuatrimestre, el profesorado participante volvió a reunirse para comentar lo experimentado previamente con sus alumnos y alumnas con el fin de que ello pudiera ayudar de alguna manera a los profesores que aún no habían impartido docencia y que lo hacían en el segundo cuatrimestre.

7. Se completaron una serie de encuestas elaboradas por la coordinadora en soporte web (imagen 3), para el análisis de la efectividad de la aplicación de la rúbrica de evaluación, tanto para el alumnado como para el profesorado.

8. Finalmente, se hizo una puesta en común en base a los resultados obtenidos en las encuestas y las conclusiones obtenidas de las reuniones mantenidas durante este curso académico.

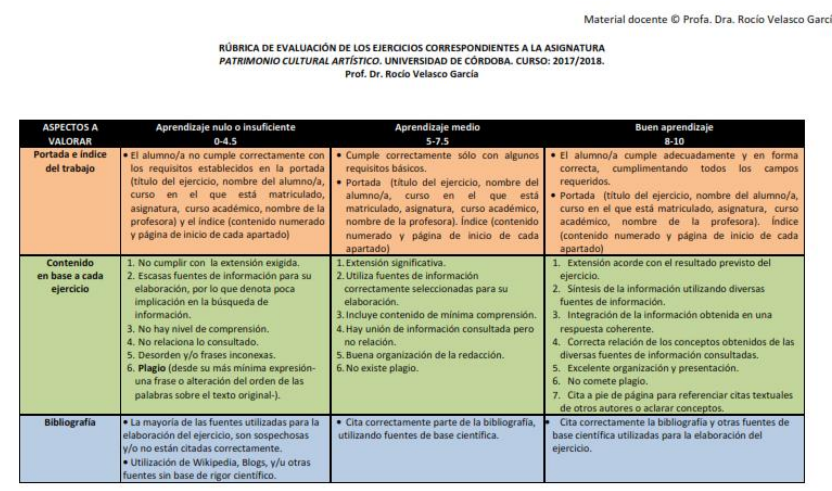

Imagen 1. Rúbrica de Evaluación. Rocío Velasco García (coordonadora del proyecto). 


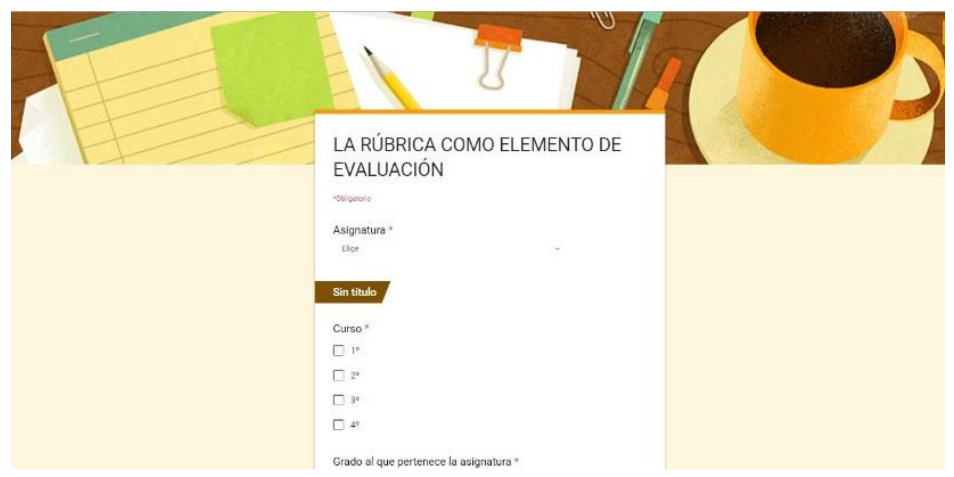

Imagen 3. Detalle de encuesta para el alumnado.

Elaboración propia de la coordinadora del proyecto en Google Docs.

\section{RESULTADOS OBTENIDOS}

Al finalizar los procesos de evaluación de las distintas herramientas establecidas en las Guías Docentes de cada asignatura implicada en el proyecto, se envió al profesorado responsable de cada una de ellas, una encuesta para el alumnado. No todo el alumnado implicado ha participado de la encuesta (160 respuestas), pero podemos establecer unos parámetros generales que nos acercan a los siguientes resultados. La mayoría de alumnos/as que realizaron la encuesta ronda entre los 18 y 20 años de edad, pues hay una mayor participación de alumnos de $1^{\circ}$ y $2^{\circ}$ curso. Entre ellos abunda el género femenino con un 62 , $3 \%$, mientras que el $37,7 \%$ es masculino.

El $100 \%$ de los alumnos encuestados comprendió claramente la rúbrica de evaluación (labor que hay que agradecer al profesorado responsable de cada asignatura).

Un 83\% utilizó la rúbrica para el desarrollo de los instrumentos de evaluación indicados por el profesor/a. Del resto, un $66,7 \%$ dice no haberla utilizado por no disponer de tiempo para ello, un $19 \%$ no la entendía, 9, $5 \%$ se cansó de ella y un 4, $8 \%$ vio complejidad en ella. En el caso anterior, del alumnado que sí llegó a utilizar la rúbrica, el 22, 1\% solo recurrió a ella en una ocasión; el $38,4 \%$, menos de 5 , el $14 \%$ más de 5 veces y el $26,7 \%$ recurrió a ella constantemente.

Hay un total de $64 \%$ del alumnado consideró que le ha resultado útil para la elaboración de los instrumentos de evaluación. Sólo el 8,5\% opinó lo contrario. Y un alto porcentaje llegó a indicar que le ha resultado una herramienta útil para conocer los criterios con los que iba a ser evaluado (más del 74,5\%). De hecho, el 87,7\% fue consciente de que debía detenerse y mejorar determinados aspectos de su formación, aunque también encontramos un 12, 3\% que opinó todo lo contrario.

Respecto al profesorado implicado en el proyecto, con una participación en la encuesta de 9 sobre 16 docentes, el 66,7\% de los docentes oyeron hablar de la rúbrica de evaluación, pero hay un total del 88, 9 \% que nunca la había utilizado anteriormente. Hay que decir que no todo el profesorado llegó a realizar la encuesta, pero sí participó de las reuniones y foros desarrollados durante todo el curso académico.

A un 66,7\% del profesorado le resultó útil esta herramienta de evaluación, mientras que a un 33, 3\% optó por indicar "regular". No constó que hubiera ningún docente participante en este proyecto al que no le resultara nada útil la aplicación de dicha herramienta. Casi la mitad del profesorado detectó una mejoría en el proceso de aprendizaje su alumnado, e innegablemente, según los resultados, el 100\% del profesorado opinó que las calificaciones finales fueron superiores que las de otros años.

Según recoge los resultados de la encuesta, la opinión del profesorado sobre su experiencia en la aplicación de la rúbrica como instrumento de evaluación en asignaturas de disciplinas humanísticas fue muy variada y enriquecedora para el trabajo colaborativo. La oponión resultante del profesorado participante del proyecto podría resumirse en que la rúbrica de evaluación supone una herramienta de gran utilidad para los procesos de evaluación en el sistema educativo universitario. En la práctica y par el profesorado participante, la rúbrica de evaluación supuso una herramienta que permitió a los alumnos saber en qué tienen que insistir en el aprendizaje. Igualmente fue considerada como una actividad positiva porque gracias a ella se reforzaron algunos de los contenidos claves, facilitando a los alumnos la comprensión de las expectativas y exigencias del profesor respecto a la elaboración de trabajos.

Se le preguntó al profesorado si veían posible una rúbrica de evaluación única (con ligeras modificaciones según el caso) para todos los miembros de su departamento. El 55, 6\% contestó que tal vez, mientras que un 33, $3 \%$ contestó sí y un 11, $1 \%$ que no. Es un hecho que para la mayor parte del profesorado participante el aunar criterios de evaluación es algo positivo para el proceso de aprendizaje. Sin embargo aún queda mucho por trabajar para llegar a la unificación de criterios comunes entre el profesorado. Aún hay quien opina tras la puesta en marcha de este proyecto, que pueden darse casos concretos de aspectos evaluables donde, quizás, no sean determinantes o útiles los sistemas de rúbrica. Por otro lado, hay un claro interés por establecer al menos algunos criterios comunes fundamentales, incluso aunque luego cada profesor/a pudiera introducir 
matices relativos a la especificidad de la asignatura o relativos a sus propias preferencias. Desde luego, hay puntos comunes en todas las asignaturas, tales como formato o composición y desarrollo de una actividad respecto al contenido general, lo que no quita que, de forma particular, cada rúbrica tenga/añadir unas especifidades añadir particularidades de acuerdo a las características de la misma.

\section{CONCLUSIONES}

Se llegó a difundir tanto entre el profesorado como el alumnado de distintas materias humanística, la herramienta de rúbrica de evaluación como proceso de enseñanza-aprendizaje. Hay que seguir trabajando en el desarrollo de mejores rúbricas de evaluación, para que no llegue a darse el caso de que algún alumno/a que indique que no ha encontrado utilidad en la rúbrica de evaluación. Quizás en ese caso el problema radique en la conformación y planificación de la misma.

Una de las cuestiones más interesantes respecto al alumnado es que la mayoría de ellos, con la utilización de esta herramienta e independientemente de su nivel de estudios, grado y materia, se dio cuenta de sus fortalezas y debilidades en la asignatura. Esto nos lleva a afianzar con su aplicación ese proceso de enseñanza-aprendizaje y, por consiguiente, los óptimos resultados obtenidos por el alumnado.

Para finalizar, al 77, 8\% del profesorado indicó que le gustaría continuar con el proyecto, mientras que a un 22, 2\% señaló que tal vez, siendo este curiosamente el menos participativo. También hay que decir que hubo un gran interés mostrado por varios docentes ajenos al proyecto y que han quedado a la espera de nueva convocatoria. Ello nos hace ver la necesidad de seguir trabajando en ello hasta encontrar fórmulas más adecuadas y la necesidad igualmente de utilizar herramientas como esta para establecer una mejor coordinación y comunicación entre alumnado-profesorado es evidente y hay que apostar por ella y porque su difusión también se amplíe a todos los miembros de cada departamento.

Por tanto, a partir de los resultados obtenidos de todo el proceso anteriormente descrito, podemos concluir que los objetivos planteados en un primer momento han sido alcanzados: A. Se dio a conocer la herramienta de rúbrica de evaluación así como su utilidad y ventajas de aplicación, configuración y uso práctico; B. Elaboración de una rúbrica de evalución propia de cada docente participante del proyecto, adaptada a sus necesidades docentes según la Guía de la asignatura donde se establecen las competencias de la misma. C. Evaluación positiva respecto a la participación del alumnado y profesorado implicado a través de la elaboración de encuestas on-online, que permitieron analizar y tener una visión crítica de la actividad. D. Muestra de interés por aprte del profesorado, tras la puesta en común de los resultados, por seguir implementando la rúbrica de evaluación en próximos cursos y en todas las asignaturas de las que sean coordinadores.

Como resumen de todo ello destacar que, en líneas generales, la experiencia ha sido positiva, viendo mejores resultados en las calificaciones del alumnado implicado en el proyecto, así como en la dinámica de evaluación del profesorado. Los participantes de este proyecto además han obeservado la necesidad de continuar con el estudio, adaptación y evaluación de esta herramienta, bajo el correspondiente seguimiento y coordinación, con el fin de establecer de forma definitiva criterios comunes entre las diversas disciplinas humanísticas de este centro educativo de la Universidad de Córdoba.

\section{AGRADECIMIENTOS}

Mi agradecimiento personal a todo el profesorado participante activamente en este proyecto, que se han volcado en él con interés, curiosidad, iniciativa, responsabilidad y trabajo en equipo.

\section{BIBLIOGRAFÍA}

BIGGS, J. (2005), Calidad del aprendizaje universitario, Narcea, Madrid.

CANO, E. (2011), Buenas prácticas en la evaluación de competencias: cinco casos de educación superior, Laertes, Barcelona.

FERNÁNDEZ, A. (2010), "La evaluación orientada al aprendizaje en un modelo de formación por competencias en la educación universitaria", Revista de Docencia Universitaria, Vol. 8, pp. 11-34.

ZAZUETA HERNÁNDEZ, María Alejandra y HERRERA LÓPEZ, Luis Fernando (2008), "Rúbrica o matriz de valoración, herramienta de evaluación formativa y sumativa”, Quaderns Digitals, no 55. 